\title{
Analysis of Trabecular Bone Microstructure Using Contour Tree Connectivity
}

\author{
Dogu Baran Aydogan ${ }^{1,2}$, Niko Moritz ${ }^{3}$, Hannu T. Aro ${ }^{3}$, and Jari Hyttinen ${ }^{1,2}$ \\ 1 Department of Electronics and Communications Engineering, \\ Tampere University of Technology, Tampere, Finland \\ ${ }^{2}$ BioMediTech, Tampere, Finland \\ 3 Orthopaedic Research Unit, Department of Orthopaedic Surgery and \\ Traumatology, Turku University Hospital and University of Turku, Turku, Finland
}

\begin{abstract}
Millions of people worldwide suffer from fragility fractures, which cause significant morbidity, financial costs and even mortality. The gold standard to quantify structural properties of trabecular bone is based on the morphometric parameters obtained from $\mu \mathrm{CT}$ images of clinical bone biopsy specimens. The currently used image processing approaches are not able to fully explain the variation in bone strength. In this study, we introduce the contour tree connectivity (CTC) as a novel morphometric parameter to study trabecular bone quality. With CTC, we calculate a new connectivity measure for trabecular bone by using contour tree representation of binary images and algebraic graph theory. To test our approach, we use trabecular bone biopsies obtained from 55 female patients. We study the correlation of CTC with biomechanical test results as well as other morphometric parameters obtained from $\mu \mathrm{CT}$. The results based on our dataset show that CTC is the $3^{\text {rd }}$ best predictive feature of ultimate bone strength after bone volume fraction and degree of anisotropy.
\end{abstract}

Keywords: trabecular bone, microstructure, binary morphology, CT imaging, connectivity, contour tree, algebraic graph theory.

\section{Introduction}

Fractures affect millions of people worldwide, causing significant morbidity, financial costs and even mortality. Several studies suggest that currently used standard diagnosis approach which is based on BMD is a limited predictor of fracture risks [1]. On the other hand, there is increasing evidence on other factors, most important one being the bone quality, which combines both structural and material properties of trabecular bone tissue [2]. Currently, the gold standard to study trabecular bone microstructure is based on morphometric parameters obtained from $\mu \mathrm{CT}$ images. Therefore, it is crucial to develop effective image processing methods that predict bone strength by analyzing $\mu \mathrm{CT}$ images.

Connectivity has long been recognized as an important parameter to predict the mechanical properties of trabecular bone [3]. The most commonly used connectivity measure is the connectivity density (Conn.D), which is based on the 
Euler number $(\chi)$ 4. For a 3D binary image that is composed of a single connected component with no holes, $1-\chi$ gives the number of tunnels that exist in the connected component. The tunnels in the case of trabecular bone are mostly associated with trabeculae. This makes Conn.D, which is defined as the number of tunnels per unit volume, an elegant tool to study trabecular bone topology. On the other hand, for most practical purposes, topology alone is not sufficient to describe connectivity properties. For example, although Conn.D includes information regarding the number of trabeculae, it does not provide information about the thickness of the connections nor the gaps between them.

In this study, we propose to use the contour tree connectivity (CTC) as an alternative connectivity measure to predict strength of trabecular bone. In [5], contour trees are utilized to provide graph representations of binary images. This representation of images transforms complex 3D data, such as the trabecular bone, into simple graphs. Based on a similar representation, CTC calculates the connectivity of binary images by calculating the connectivity of graphs.

This study introduces CTC as a novel morphometric parameter to study bone quality. For that, we study bone biopsies of 55 patients and show how CTC can be used to predict the strength of trabecular bone.

\section{Methods and Materials}

\subsection{Contour Tree Connectivity (CTC)}

Representation of Binary Images Using Contour Trees: Contour tree is a topological abstraction that follows the evolution of level sets as they appear, join, split or disappear by means of a graph. In image processing, it has been mostly used for volume simplification and visualization of high dimensional scalar fields [6]. Contour trees are first used for binary images in [5], in order to develop a graph representation, which takes into account both the geometrical properties of foreground object(s) and how the object(s) is/are placed on the background. This is done by first applying the Euclidean distance transform (EDT) and than estimating the contour tree of the transformed image. An example on a small crop of a $2 \mathrm{D}$ trabecular bone slice is shown in Fig 1 .

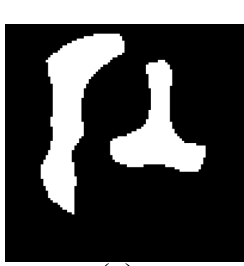

(a)

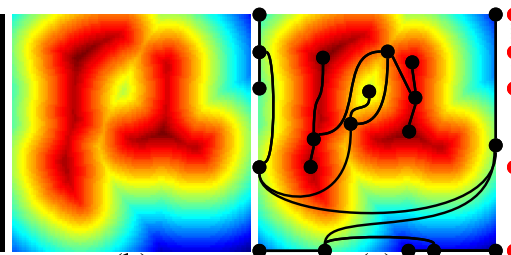

(b)

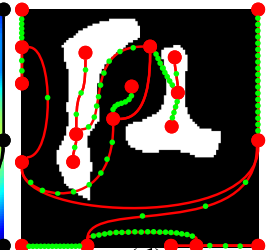

(d)

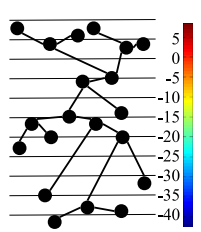

(e)

Fig. 1. (a) Input image (b) EDT of input (c) Contour tree drawn on top the EDT image (d) Supplemented contour tree with red nodes from the contour tree and green nodes from supplementation (e) Contour tree with level lines and EDT colormap. 
Extraction Steps and the Algorithms Used to Compute CTC: The main idea of CTC is to compute the structural connectivity of a binary image by computing the connectivity of its contour tree representation. Fig 2 shows the flowchart for extracting the CTC feature.

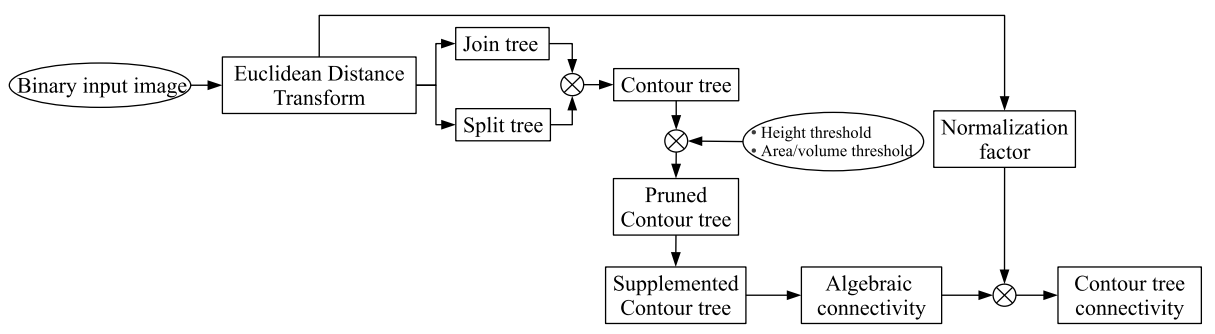

Fig. 2. Flowchart for the CTC feature extraction. From a binary input image, firstly EDT, then the supplemented contour tree (SCT) and finally the CTC is calculated as the normalized algebraic connectivity of SCT.

Starting with a binary image, we first create a real valued image using EDT. With a signed EDT, we transform the foreground to positive and background to negative real numbers with their distances to the nearest non-object pixels. The EDT image is used as the input for computing the contour tree.

We picked a popular method called the sweep and merge to compute the contour tree [7. The sweep and merge method starts with computing the join and split trees; in a later stage, these are merged to form the contour tree. Join and splits trees represent the connectivities of upper and lower level sets respectively.

In order to remove the noise from the graph, we perform basic pruning operations on the contour tree. In order to prune edges or vertices, two user defined inputs are utilized. Edges to be removed must have less area/volume than the defined area/volume threshold and the vertices on each sides of the edge should have a level difference below the given level threshold.

As the last graph operation, we create a new tree by introducing integer valued levels in between the critical points. This final tree is called the supplemented contour tree (SCT). SCT simply shows how foreground/background regions in the image are positioned by the number of vertices along edges. The example in Fig. [1 shows the difference between the contour tree and the SCT.

Graph Spectrum and Algebraic Connectivity: CTC measures the connectivity of a binary image indirectly by computing the connectivity of its contour tree representation. To achieve this, we use algebraic graph theory and spectrum of graphs. The spectrum of a graph $\mathcal{G}$ is the set of the eigenvalues of its Laplacian matrix that is given as $L(\mathcal{G})=D(\mathcal{G})-A(\mathcal{G})$. Here $D(\mathcal{G})$ and $A(\mathcal{G})$ are the degree diagonal and the adjacency matrices respectively. The eigenvalues of the Laplacian matrix can be sorted as $0=\lambda_{0} \leq \lambda_{1} \leq \lambda_{2} \leq \ldots \leq \lambda_{n}$ for any connected graph. Since $\lambda_{1}=0$ for disconnected graphs, Fiedler named the second smallest eigenvalue, $\lambda_{1}$, as algebraic connectivity. 
From the definition of algebraic connectivity, for all SCT, $\lambda_{1}>0$ is a lower bound. Also for all SCT, $\lambda_{1} \leq 2\left(1-\cos \frac{\pi}{h+1}\right)$ is an upper bound, where $h$ is the height of SCT. (The upper bound follows from Theorem 6.4.1 (page 240) and Corollary 6.4.3 (page 242) given in [8].)

Among the set of trees with same height, trees with wider horizontal spread of vertices have lower $\lambda_{1}$. Therefore, we define CTC of a binary image $I$ by normalizing $\lambda_{1}$ with as follows:

$$
C T C=\frac{\lambda_{1}(\mathcal{G})}{2\left[1-\cos \left(\frac{\pi}{\left\lfloor l_{\text {max }}^{g}\right\rceil-\left\lfloor l_{\text {min }}^{g}\right\rceil+1}\right)\right]}
$$

Here $\mathcal{G}$ is the SCT of $I . \lambda_{1}$ is the algebraic connectivity of $\mathcal{G} . l_{\text {max }}^{g}$ and $l_{\text {min }}^{g}$ are the global max and min of EDT of $I$ and $\lfloor$.$\rceil is the rounding operator.$

From Equation 1, it follows that for any binary image, $0<C T C \leq 1$.

\subsection{Patient Data, $\mu \mathrm{CT}$ Imaging and Biomechanical Testing}

The original study population consisted of 61 patients with primary hip osteoarthritis who underwent primary cementless total hip arthroplasty (THA). The demographic data of the patients was described previously in [9]. During THA surgery, a cancellous biopsy was taken from the intertrochanteric region of the proximal femur as described earlier in [10]. The biopsy specimen were wrapped in saline-wet sponges and placed in a sealed plastic bag and stored frozen at $-20^{\circ} \mathrm{C}$ until machining. Multiple specimens were prepared from each biopsy under saline irrigation using a high-speed trephine drill. The cylindrical specimens were scanned with $\mu \mathrm{CT}$ (SkyScan 1072, Kontich, Belgium) with isotropic voxel resolution of $16.3 \mu \mathrm{m}$.

From the $\mu \mathrm{CT}$ images, 10 parameters were calculated. These are: bone volume fraction (BV/TV), bone mineral density (BMD), mean trabecular thickness (Tb.Th), trabecular seperation (Tb.Sp), trabecular number (Tb.N), trabecular bone pattern factor (Tb.Pf), structure model index (SMI), degree of anisotropy (DA), Conn.D and CTC. Conn.D and CTC were calculated using MATLAB, whereas the other parameters were calculated using CTan software (SkyScan). For each patient, the mean results of the parameters obtained from multiple specimens were used in the study.

Following the $\mu \mathrm{CT}$ imaging, the specimens were used in compression testing to failure using a universal mechanical testing device (Avalon Technologies, Rochester, MI, USA). Tests were performed at a constant speed of 0.825 $\mathrm{mm} / \mathrm{min}$ and the load data were continuously recorded by the data acquisition system (Visual Designer, Intelligent Instrumentation, Tucson, AZ, USA). Values for the ultimate tensile strength $\left(\sigma_{U}\right)$ were calculated from the raw data files using Origin software (Origin Lab Corp. Northampton, MA, USA).

The analyses were performed on 55 patients out of 61 . Six patients were excluded due to incomplete data. The patients were divided into three groups with respect to their trabecular bones' ultimate strength. The first group, G-1, 
has $\sigma_{U} \leq 0.35 \mathrm{MPa}$, the second group, G-2, has $0.35 \mathrm{MPa}<\sigma_{U} \leq 1 \mathrm{MPa}$ and the last group, G-3, has $\sigma_{U}>1 \mathrm{MPa}$. The thresholds were chosen manually by taking into account the jumps in $\sigma_{U}$. According to this division, 26 patients belong to G-1, 20 patients belong to G-2 and 9 patients belong to G-3. Fig 3 shows the values for the ultimate tensile strength in ascending order and the threshold levels used to group the patients.

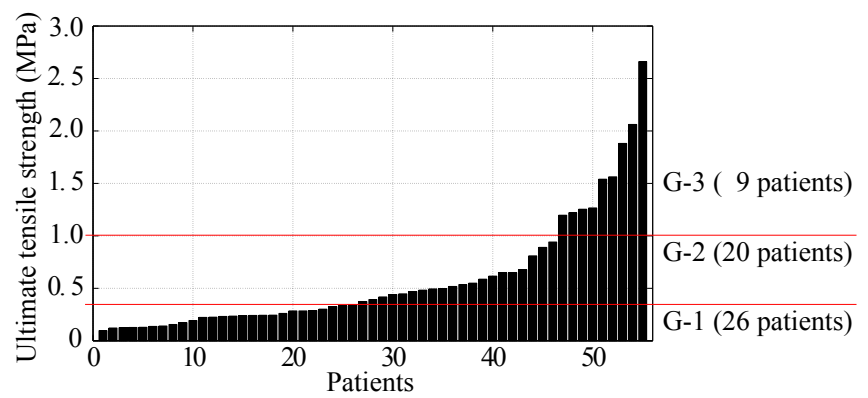

Fig. 3. Ultimate strength values obtained from the measurements in ascending order. Red lines show the thresholds that are used to categorize the patients into three groups.

\subsection{Regression Analysis, Feature Selection and Classification Method}

The correlation between ultimate tensile strength and BV/TV was reported earlier in [10]. In this work, we study the other correlations and calculate the coefficient of determination $\left(R^{2}\right)$ between $(a) \sigma_{U}$ and $\mu \mathrm{CT}$ parameters $(b) \mathrm{BV} / \mathrm{TV}$ and $\mu \mathrm{CT}$ parameters (c) $\mathrm{CTC}$ and $\mu \mathrm{CT}$ parameters.

To identify the most predictive $\mu \mathrm{CT}$ parameters for ultimate tensile strength, we used the data from all the patients and did a sequential forward feature selection (SFS). SFS selects a subset of $\mu \mathrm{CT}$ features that best predict the $\sigma_{U}$ measurements by sequentially adding features. Each feature is selected after a repeated application of 10 -fold cross validation. Among all candidates, the feature set that gives the minimum mean deviance is picked. Deviance measures the quality of the fit as the residual sum of squares obtained from generalized linear model regression. We used SFS on two sets of features. The first feature set, Case- 1 , had all the $10 \mu \mathrm{CT}$ parameters. The second feature set, Case-2, did not have CTC but had all the other 9 parameters.

Lastly, we used linear discriminant analysis for classifications. For this, we used 13, 10 and 4 patients from groups G-1, G-2 and G-3 respectively as the training set. The rest of the 28 patients are used in the tests. For both feature sets, Case-1 and Case-2, we sequentially classified test subjects into groups by increasing the number of significant features. 


\section{Results}

\subsection{Demonstrative CTC Study}

We picked three $\mu \mathrm{CT}$ images to show example CTC values for different cases. Fig 4 shows these samples, corresponding ultimate tensile strength, BV/TV and CTC values. Since the actual contour trees contain several hundreds of vertices, simplified versions obtained from the crops are plotted below each $\mu \mathrm{CT}$ image.

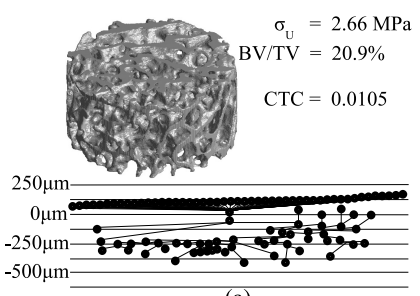

(a)

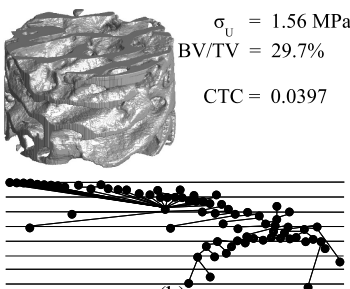

(b)

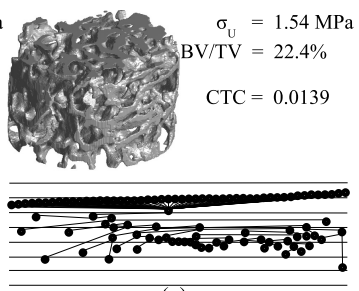

(c)

Fig. 4. Example $\mu \mathrm{CT}$ images and corresponding $\sigma_{U}, \mathrm{BV} / \mathrm{TV}$ and $\mathrm{CTC}$ values. Below each sample is a simplified version of the contour tree. (a) shows the strongest bone in the dataset, (b) has the highest BV/TV in the dataset, (c) has a similar $\sigma_{U}$ value to the one in (b) despite less BV/TV.

\subsection{Correlation Analysis}

Fig 5 shows the calculated $R^{2}$ values. From left to right, Fig 5 shows the linear correlation between (a) $\sigma_{U}$ and $\mu \mathrm{CT}$ parameters (b) BV/TV and $\mu \mathrm{CT}$ parameters (c) CTC and $\mu \mathrm{CT}$ parameters.

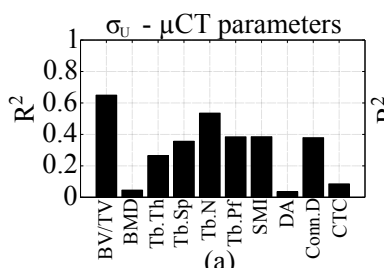

(a)

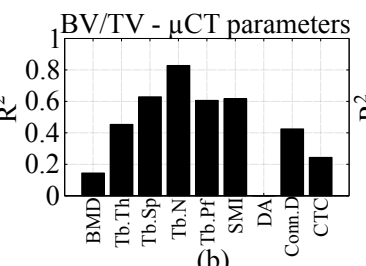

(b)

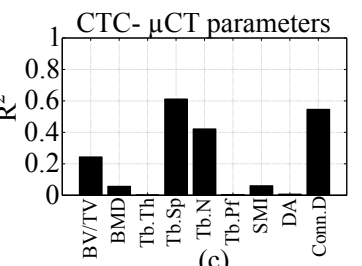

(c)

Fig. 5. Linear regression analyses show (a) $\sigma_{U}$ is best correlated with BV/TV (b) Tb.N, Tb.Sp, SMI and Tb.Pf are correlated with BV/TV (c) CTC is uncorrelated with $\mu \mathrm{CT}$ parameters other than Tb.Sp, Conn.D and Tb.N.

\subsection{Feature Selection and Classification Results}

Table 1 1 shows the order of the selected features for Case-1 and Case-2 using SFS and the classification accuracies. 
Table 1. The order of the selected features that best predict $\sigma_{U}$ measurements and the classification accuracies using feature sets Case- 1 and Case-2. CTC is chosen as the $3^{\text {rd }}$ best feature by SFS. BV/TV and DA are the top two features.

\begin{tabular}{|c|c|c|c|c|c|c|c|c|c|c|}
\hline & $1^{\text {st }}$ & $2^{n d}$ & $3^{\text {rd }}$ & $4^{t h}$ & $5^{t h}$ & $6^{t h}$ & $7^{t h}$ & $8^{t h}$ & $9^{t h}$ & $10^{t h}$ \\
\hline & & $\begin{array}{l}\text { DA } \\
50 \%\end{array}$ & & & & & & & & $\begin{array}{l}\text { BMD } \\
67.9 \%\end{array}$ \\
\hline & $\begin{array}{c}\mathrm{BV} / \mathrm{TV} \\
60.7 \%\end{array}$ & $\begin{array}{c}\text { DA } \\
50 \%\end{array}$ & $\begin{array}{c}\text { SMI } \\
60.7 \%\end{array}$ & $\begin{array}{c}\text { Conn. D } \\
60.7 \%\end{array}$ & & & & & $57.1 \%$ & - \\
\hline
\end{tabular}

\section{Discussion and Conclusion}

Traditionally, the connectivity of trabecular bone has been studied from a topological point of view using the Euler number. This interpretation has been linked to the mechanical properties of bone 4. With a structural interpretation, CTC provides new connectivity information that takes into account the thickness of the trabeculae as well as their separation.

Fig 4 shows example CTC values obtained for $3 \mu \mathrm{CT}$ images. Generally an increased number of trabeculae decreases CTC by two mechanisms. Firstly, trabeculae appear as local maximas in the EDT image, which introduces vertices into the join tree. Secondly, gaps between trabeculae, that appear as local minimas, introduce vertices into the split tree. As a result, a sample with a large number of trabaculae is represented with a spread of several vertices above and below 0 level. In the contour trees shown in Fig, 4 , vertices at higher levels correspond to thicker trabeculae, whereas lower levels correspond to larger gaps between trabeculae. By quantifying the connectivity of this representation, CTC quantifies the connectivity of trabeculae with regard to their thickness and separation.

Fig 4 also shows how CTC complements other features such as BV/TV. From Fig 4 a to Fig 4b, there is a $1.1 \mathrm{MPa}$ decrease in $\sigma_{U}$ despite the $8.8 \%$ increase in BV/TV. Additionally, from Fig 4b to Fig 4c, the $7.3 \%$ decrease in BV/TV does not change $\sigma_{U}$ significantly. The contour tree for Fig $4 \mathrm{~b}$ with few trabeculae and large gaps accounts for these differences. This compact tree representation yields a large CTC value, which indicates structural weakness for this sample.

The correlation analysis in Fig 5 a shows that BV/TV alone explains more than $65 \%$ of the variance in $\sigma_{U}$. Second most correlated feature with $\sigma_{U}$ is Tb.N. Fig 5 b shows that, Tb.N, Tb.Sp, SMI and Tb.Pf are correlated with BV/TV. On the other hand, CTC is uncorrelated with most parameters.

Table 1 shows that CTC is the $3^{\text {rd }}$ best predictive feature of ultimate strength after BV/TV and DA. This coincides with both earlier and recent studies where $\mathrm{BV} / \mathrm{TV}$ and DA are shown to be good predictors for $\sigma_{U}$ [11]12]. Interestingly, our results show that BMD is neither correlated with $\sigma_{U}$ nor it is significant when predicting $\sigma_{U}$. It is observed from the correlation and classification results that most of the commonly used parameters are redundant. For Case-2, once BV/TV is used, the inclusion of many features does not improve accuracy. Moreover, 
additional features decrease the accuracy more for Case- 2 than Case- 1 due to the curse of dimensionality. Lastly, it is observed that 3 subsets of Case- 1 that include CTC reach an accuracy of $71.4 \%$, which could not be achieved by any Case-2 subsets.

The application of linear regression for correlation analysis has the limitation that only linear relations are investigated. LDA also suffers from the same problem regardless of our use of independent traning and test sets. Further investigations that include non-linear relations might improve the results. Additionally, in order to generalize the results, a wider spectrum of patients need to be studied.

In this article, we introduce the contour tree connectivity (CTC) to predict the ultimate strength of trabecular bone. Our results show that most morphometric parameters do not provide additional information on top of bone volume fraction. CTC on the other hand, is uncorrelated with bone volume fraction and most other parameters. Our findings show that CTC is the $3^{r d}$ best predictive parameter for ultimate strength after bone volume fraction and degree of anisotropy. By providing new structural connectivity information, CTC offers more options to better predict bone quality.

\section{References}

1. Heaney, R.: Is the paradigm shifting? Bone 33(4), 457-465 (2003)

2. Bouxsein, M.L.: Bone quality: where do we go from here? Osteoporosis International 14, 118-127 (2003)

3. Fields, A., Keaveny, T.: Trabecular architecture and vertebral fragility in osteoporosis. Current Osteoporosis Reports 10(2), 132-140 (2012)

4. Odgaard, A., Gundersen, H.J.G.: Quantification of connectivity in cancellous bone, with special emphasis on 3-D reconstructions. Bone 14(2), 173-182 (1993)

5. Aydogan, D.B., Hyttinen, J.: Binary image representation by contour trees. In: Proc. SPIE 8214, Medical Imaging 2012: Image Processing, 83142X (February 2012)

6. Carr, H., Snoeyink, J., Van De Panne, M.: Flexible isosurfaces: Simplifying and displaying scalar topology using the contour tree. Computational Geometry: Theory and Applications 43(1), 42-58 (2010)

7. Carr, H.: Topological Manipulation of Isosurfaces. PhD thesis, Department of Computer Science, The University of British Columbia (2004)

8. Molitierno, J.J.: Applications of Combinatorial Matrix Theory to Laplacian Matrices of Graphs. Taylor \& Francis (2012)

9. Mäkinen, T.J., Alm, J.J., Laine, H., Svedström, E., Aro, H.T.: The incidence of osteopenia and osteoporosis in women with hip osteoarthritis scheduled for cementless total joint replacement. Bone 40(4), 1041-1047 (2007)

10. Moritz, N., Alm, J.J., Lankinen, P., Mäkinen, T.J., Mattila, K., Aro, H.T.: Quality of intertrochanteric cancellous bone as predictor of femoral stem rsa migration in cementless total hip arthroplasty. Journal of Biomechanics 44(2), 221-227 (2011)

11. Goulet, R.W., Goldstein, S.A., Ciarelli, M.J., Kuhn, J.L., Brown, M.B., Feldkamp, L.A.: The relationship between the structural and orthogonal compressive properties of trabecular bone. Journal of Biomechanics 27(4), 375-389 (1994)

12. Tassani, S., Öhman, C., Baleani, M., Baruffaldi, F., Viceconti, M.: Anisotropy and inhomogeneity of the trabecular structure can describe the mechanical strength of osteoarthritic cancellous bone. Journal of Biomechanics 43(6), 1160-1166 (2010) 